\title{
Evidence of Antidepressive Effects of a Wakan-yaku, Hochuekkito, in Depression Model Mice with Learned-Helplessness Behavior
}

\author{
Michihisa Tohda ${ }^{1,2}$ and Salin Mingmalairak ${ }^{1}$ \\ ${ }^{1}$ Division of Medicinal Pharmacology, Institute of Natural Medicine, University of Toyama, Toyama 930-0194, Japan \\ ${ }^{2}$ Wakan-yaku Theory-Based Integrated Pharmacology, Graduate School of Innovative Life Science, University of Toyama, \\ Toyama 930-0194, Japan
}

Correspondence should be addressed to Michihisa Tohda; tohdam@inm.u-toyama.ac.jp

Received 23 September 2013; Revised 2 December 2013; Accepted 5 December 2013

Academic Editor: Han Chae

Copyright (C) 2013 M. Tohda and S. Mingmalairak. This is an open access article distributed under the Creative Commons Attribution License, which permits unrestricted use, distribution, and reproduction in any medium, provided the original work is properly cited.

\begin{abstract}
Wakan-yaku is a type of Japanese and Sino traditional, systematized medical care that has been practiced for hundreds of years. This medicinal system includes many antidepressive prescriptions. One of the candidates is Hochuekkito, although experimental evidence has not yet been established clearly. To obtain evidence, a depression model of learned-helplessness (LH) mice was used. Based on the score of escape failure, an index of the depression degree, mice with a depressive condition were selected to assess Hochuekkito's effects. This selection was significant and effective in the following two points: evaluation of the drug effect under disease conditions and minimization of the number of animals. Treatment with Hochuekkito ( 1 and $5 \mathrm{~g} / \mathrm{kg}$ p.o.; estimated galenical amount) for 14 days significantly decreased the depression index, the number of escape failures, and desipramine (10 mg/kg p.o.) suggesting that Hochuekkito has an antidepressive effect.
\end{abstract}

\section{Introduction}

Since the discovery of the antidepressive effect of imipramine in the 1950s, clinical antidepressants have been developed with monoamine transporters as the target molecules [1]. Meanwhile, many "Wakan-yaku prescriptions" have been traditionally used to treat depression. By studying the action mechanism of these Wakan-yaku prescriptions, elucidation of the generation mechanism of depression and the development of new antidepressants are expected; however, there is little experimental evidence for the antidepressive effects of Wakan-yaku.

The learned-helplessness (LH) behavior of experimental animals caused by inescapable footshocks is one behavioral phenotype relevant to depression $[1,2]$. Since this behavior can be ameliorated by antidepressants, LH animals have been used for studying the pathophysiology of depression and the actions of antidepressants $[3,4]$. LH model animals can evaluate a drug effect under more pathological conditions than the forced swimming test and tail suspension test, which are evaluated by correlation. It has been also reported that the forced swimming itself is a strong and sustained stress [5]. Therefore, in this study, the effect of a Wakan-yaku prescription, Hochuekkito, was evaluated using LH mice.

Hochuekkito has antidepressant-like effects in the forced swimming test [6]. A description of Hochuekkito (Bu Zhong Yi Qi Tang in Chinese) first appeared in Neiwaishang Bianhuolun, which was written by Dong Yuan Li in 1247. At that time, people living around Li had lost their physical strength because of malnutrition during war; therefore, $\mathrm{Li}$ developed Hochuekkito for the purpose of "improvement of the digestive system ("hochu" in Japanese) and promoting vigor ("ekki")" [7]. Then Hochuekkito was sometimes used for the treatment of depressive symptoms with a lack of appetite in Kanpo-traditional medicines. Antidepressants cause a lack of appetite as a side effect. Hochuekkito seems to be desirable to treat depression with a lack of appetite; however, there is little evidence for the antidepressant action of Hochuekkito, 
with the result that it is rarely used clinically; therefore, we examined the antidepressant effect of Hochuekkito using the LH model.

\section{Materials and Methods}

2.1. Hochuekkito Preparation. Hochuekkito was prepared by mixing ten dried crude forms of herbal components (purchased from Tochimoto Tenkuido Co., Ltd., Osaka, Japan) and preparing an extract from the mixture as previously reported [8]. The qualitative certification was done by $3 \mathrm{D}-$ HPLC (see Supplementary Figure 1 available on line at http://dx.doi.org/10.1155/2013/319073) under the same condition in previous report [8]. In brief, $49 \mathrm{~g}$ of a ten galenical mixture was added to $500 \mathrm{~mL}$ distilled water and extracted at $100^{\circ} \mathrm{C}$ for $60 \mathrm{~min}$. Galenicals were removed while the solution was still hot. The extracted solution was filtered and freeze-dried to obtain dry extract powder. Part of the frozen extract was lyophilized to assess the yield (34\%). The dose of Hochuekkito was shown by the estimated galenical amount using this yield value. The quality of this drug is controlled by measuring the contents by high-performance liquid chromatography (HPLC) (data not shown). Before the HET extract was applied to cultured cells, it was centrifuged to remove insoluble matter and filter-sterilized.

2.2. Animals. Ten-week-old male ICR mice (Japan SLC Inc., Shizuoka, Japan) weighing 38-40 g were housed in groups of eight per cage under standard conditions: $25 \pm 1^{\circ} \mathrm{C}$ ambient temperature; $65 \pm 5 \%$ relative humidity; $12 \mathrm{~h}$ light/dark cycle (lights on from 07:30 to 19:30). They had ad libitum access to food and water and were allowed to acclimatize to these housing conditions for 1 week prior to the experiments. Care and use of the animals was in accordance with the Guiding Principles for the Care and Use of Animals in the Field of Physiological Science of the Physiological Society of Japan and the Institutional Animal Use and Care Committee of University of Toyama. Studies were designed to minimize pain and the number of animals used.

2.3. Learned-Helplessness Paradigm. The paradigm and experimental conditions used to induce LH behavior in mice are the same as previously reported [9]. In the avoidance test conducted on day 4 , mice were selected for the experiments as $\mathrm{LH}$ animals if escape failed more than 10 times.

2.4. Drug Administration. All drugs were dissolved and diluted with water to the appropriate concentration. Hochuekkito at doses of 1 or $5 \mathrm{~g} / \mathrm{kg}$ and desipramine (SigmaAldrich, St. Louis, MO, USA) at a dose of $10 \mathrm{mg} / \mathrm{kg}$ were administered orally through a feeding needle inserted down the throat of the mice in a volume of $10 \mathrm{~mL} / \mathrm{kg}$ body weight. Drug was administered once daily for 14 days, starting on day 5 . On day 18 , the mice were tested for escape ability under escapable shock conditions $60 \mathrm{~min}$ after the last administration of drug.

2.5. Statistical Analysis. All data are presented as mean \pm S.E.M. Two-way repeated-measure analysis of variance

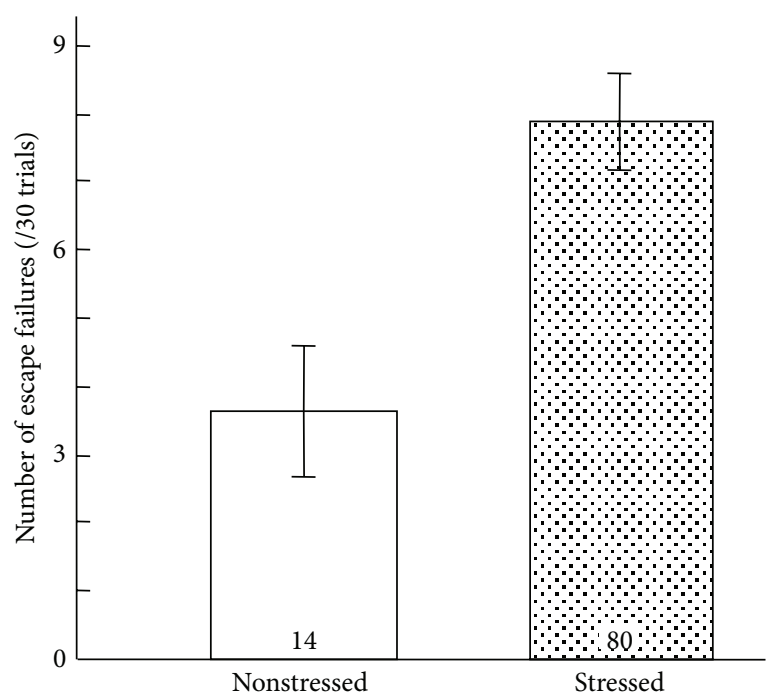

FIGURE 1: Learned-helplessness paradigm to develop depression model mice. The number of escape failures of the footshock-naive control (nonstressed) and animals that had received inescapable footshock (stressed) was analyzed. The stressed animals received footshock under inescapable conditions once a day for 3 consecutive days. The avoidance test was performed on days 4 and 18 under escapable conditions. The avoidance test on day 18 was conducted $60 \mathrm{~min}$ after the last drug administration. The data are expressed as the mean \pm S.E.M.

(ANOVA), followed by the post hoc analysis test, was used for the statistic evaluation of behavioral data obtained from the avoidance test.

\section{Results}

As shown in Figure 1, the failure scores of the animals exposed to inescapable footshock stress $(7.875 \pm 0.714, n=80)$ were higher than those of naïve control animals $(3.642 \pm 0.941$, $n=14$ ); however, the difference between these values was not enough to easily evaluate the effect of drugs. Many animals seem to be required for evaluation under this experimental condition. In addition, the results of LH animals did not seem to show normal distribution: normal animals and those with depression seemed to coexist (Figure 2). Therefore, the animals were divided into LH mice (depression model) and non-LH (depression-resistant model) based on the escape failure scores at the end of the avoidance test on day 4 (Figure 2). Out of the animals that had been exposed to inescapable footshock on day 1 , around $30 \%$ of the animals exhibited LH behavior in the avoidance test conducted on day 4 (Figure 2). This result was consistent with previous reports $[10,11]$. The LH animals were then randomly divided into 4 groups. Each group received oral administration of 1 or $5 \mathrm{~g} / \mathrm{kg}$ Hochuekkito, $10 \mathrm{mg} / \mathrm{kg}$ desipramine, or water as vehicle once daily for 14 days from day 5 . As shown in Figure 3, when vehicle-treated $\mathrm{LH}$ animals were subjected to footshock under an escapable condition on day 18, they exhibited a deficit in escape performance. On the other hand, desipramine $(10 \mathrm{mg} / \mathrm{kg} /$ day $)$, a typical antidepressant, 


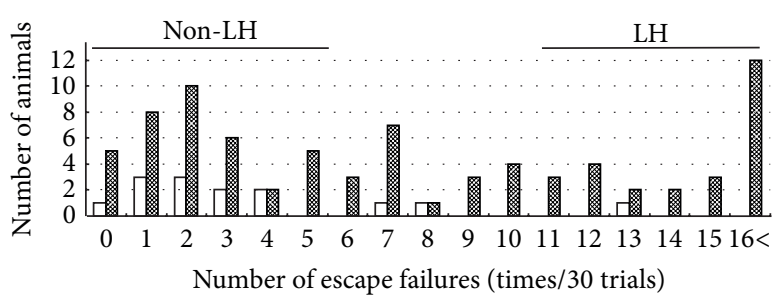

Figure 2: Phenotypic difference of mice in LH behavior test. The number of escape failures by the footshock-naïve control (open bars) and animals that had received inescapable footshock (closed bars) was analyzed. This diagram shows the distribution of the number of failures in this avoidance test. Animals that had received inescapable footshocks in the training session were classified into two groups: the LH group with failure scores no less than 10 as depressive mice and the non-LH group with failure scores no more than 5 as stressresistant mice.

significantly decreased the number of escape failures in post hoc analysis $(P=0.002)$. This significant difference was obtained by the data from only five animals each group. These results suggest that this method to evaluate the antidepressant effect using depressed animals has high sensitivity and can assess the effect with the minimum number of animals. Effects of Hochuekkito ( 1 and $5 \mathrm{~g} / \mathrm{kg} /$ day) also showed a significant difference versus vehicle-treated $\mathrm{LH}$ animals in post hoc analysis $(1 \mathrm{~g} / \mathrm{kg} /$ day: $P=0.014,5 \mathrm{~g} / \mathrm{kg} /$ day: $P=$ 0.027 ), although the number of escape failures did not improve (Figure 3).

\section{Discussion}

This report suggests the importance of using depression model animals to evaluate drugs for depression. These model animals, represented by LH mice in this study, could be divided into animals with and without depression using this behavioral pharmacological method. The number of animals with depression could be increased by applying higher strength electrical stimulation, but this is unfavorable from the viewpoint of animal welfare. Around 30\% of animals showed depression symptoms under the conditions of this experiment. Only animals in a depressed condition were given drugs to evaluate the antidepressant effect.

The "general pharmacological order" to evaluate the effects of drugs and/or treatments using all of the animals in the group is inefficient. In the general pharmacological order manner of this LH method, the difference in escape failure score between stressed and nonstressed mice was small (7.9 versus 3.6) (Figure 1), and it was doubtful whether the score showed normal distribution (Figure 2). Many animals are necessary for drug evaluation. Since medicines are used for disease treatment, it is logical to use only for experiments animals that have the disorder being evaluated by experimental diagnostics. Indeed in this experiment, drug evaluation was possible using the minimum number of animals efficiently by choosing depression conditioned animals; five in each group was sufficient. Desipramine, a typical antidepressant, improved the index of depression. We previously reported the

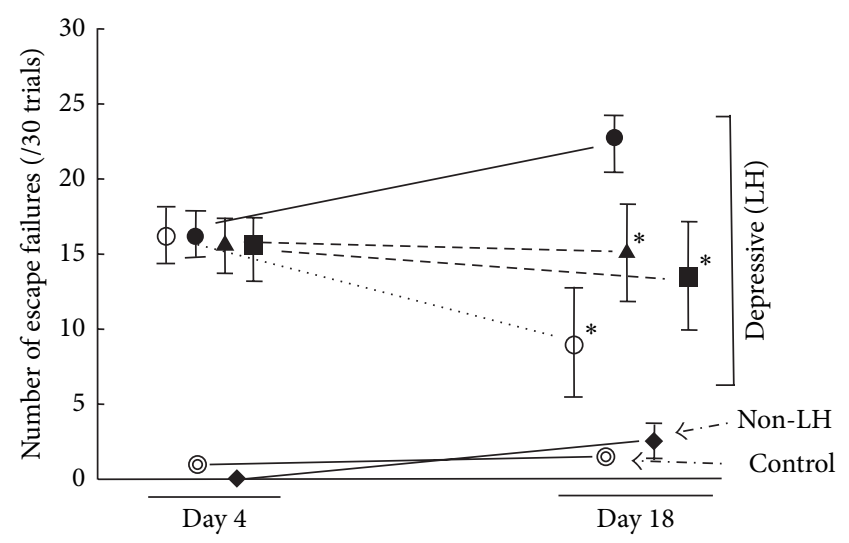

Figure 3: Effects of Hochuekkito and desipramine in the LH behavior test using depressive conditioned mice, non-LH mice and naïve control mice. The animals, except for naïve control (๑), received inescapable footshock once a day for 3 consecutive days. The avoidance test was performed on day 4 and 18 under escapable conditions. One g/kg/day Hochuekkito (४), $5 \mathrm{~g} / \mathrm{kg} /$ day Hochuekkito $(\square), 10 \mathrm{mg} / \mathrm{kg} /$ day desipramine $(\bigcirc)$ or water as a vehicle $(\bullet)$ was p.o. administered in depressive conditioned mice once daily for 14 days from day 5. Water was p.o. administered also in non-LH condition $(\diamond)$ and in naïve control $(\odot)$. The avoidance test on day 18 was conducted $60 \mathrm{~min}$ after the last drug administration. The data are expressed as the mean \pm S.E.M. ( $n=5$ per group). Significance: ${ }^{*} P<0.05$ versus vehicle-treated mice on day 18 ( $\bullet$ ) (two-way repeated ANOVA followed by the Student-Newman-Keuls test).

similar effect of imipramine [9], whereas the index became worse in water-administered animals with depression. These results suggest that this strategy is highly useful to evaluate the drug effect using the minimal number of animals.

Based on the above-mentioned points, the effect of Hochuekkito was evaluated as an antidepressant. The results showed the antidepressive ability of Hochuekkito since the index score of depression was significantly lower than that in the water-administrated group, although the score was not improved by 14 days of treatment, as shown in desipramine. Therefore, this result may provide evidence that Hochuekkito is an antidepressant or at least prevents pathologic aggravation. A previous report that showed evidence of Hochuekkito's antidepressive potential used the forced swimming test [6]; however, it has been reported that the forced swimming itself is a strong and sustained stress [5]. In addition, the forced swimming test is based on general pharmacological order. It does not use animals with depression, and it is merely a convincing rating system based on correlation. This experimental method reflecting depression is useful and valuable to obtain pharmacological evidence to support Wakan-yaku prescriptions, not only Hochuekkito, and to elucidate the action mechanism and active ingredients.

It is important to use the depressed state animal for evaluation of an antidepressant effect. In addition to the $\mathrm{LH}$ model, a chronic mild stress (CMS) model animal is also used for the evaluation [12]. The selection of the depressive state animals, in also the CMS model, may allow us to get significant information under the decreased number of 
animals. It is a further important point of view for animal protection. In alternative medicine, there are many materials from which an antidepressant effect is expected. For example, the ingredients of royal ielly [13] and Perillae Herba [14] have been reported. In addition, another Wakan-yaku prescription Juzentaihoto (Shi Quan Da Bu Tang in Chinese) is also used to expect the clinically antidepressant effect in Kanpotraditional medicines. Although there are many reports about Juzentaihoto for cancer treatment and immune functions [15], the scientific evidence about the antidepressant effect is not provided yet. Both Juzentaihoto and Hochuekkito are constructed by ten kinds of crude drugs, and five kinds are the same among the ten. The roles of the common 5 of crude drugs group and the "personality" induced by the peculiar 5 of crude drugs group in the efficacies of Hochuekkito and Juzentaihoto will be also interesting. For the detailed studies such as the difference in mechanism, the examination based on this way will be an advantage. Authors have preliminary results for Hochuekkito about the stimulation to alimentary system by the peculiar 5 of crude drugs group having the prescription-specific contribution of Hochuekkito for an antidepressive effect and the inhibitory effect of prior administration of an antidepressant such as imipramine because of the downregulation of alimentary system. The detailed mechanisms of that will be a further report by using this way of experiment and the MRI diagnostics.

\section{Conflict of Interests}

The authors declare that there is no conflict of interests regarding the publication of this paper.

\section{Acknowledgment}

This work was supported by a Grant-in-Aid for Scientific Research (A) (\#21249047) from Japan Society for the Promotion of Science (JSPS) to M.T.

\section{References}

[1] E. J. Nestler, M. Barrot, R. J. DiLeone, A. J. Eisch, S. J. Gold, and L. M. Monteggia, "Neurobiology of depression," Neuron, vol. 34, no. 1, pp. 13-25, 2002.

[2] P. Willner, "Animal models of depression: validity and applications," Advances in Biochemical Psychopharmacology, vol. 49, pp. 19-41, 1995.

[3] S. Chourbaji, C. Zacher, C. Sanchis-Segura, C. Dormann, B. Vollmayr, and P. Gass, "Learned helplessness: validity and reliability of depressive-like states in mice," Brain Research Protocols, vol. 16, no. 1-3, pp. 70-78, 2005.

[4] K. Takamori, S. Yoshida, and S. Okuyama, "Availability of learned helplessness test as a model of depression compared to a forced swimming test in rats," Pharmacology, vol. 63, no. 3, pp. 147-153, 2001.

[5] J. C. Lemos, M. J. Wanat, J. S. Smith et al., "Severe stress switches CRF action in the nucleus accumbens from appetitive to aversive," Nature, vol. 490, no. 7420, pp. 402-406, 2012.

[6] H. Watanabe, K. Matsumoto, T. Satoh, H. Ohta, and H. Matsuda, "Psychopharmacological studies on Shimotsu-to,
Kakkon-to and Oren-gedoku-to using animal models," Journal of Medical and Pharmceutical Society for Wakan-Yaku, vol. 7, no. 2, pp. 99-107, 1990 (Japanese).

[7] M. Mayanagi, "The history of a Chinese herbal prescription $\mathrm{Bu}$ Zhong-Yi-Qi-Tang," Gendai Toyo Igaku, vol. 16, no. 4, pp. 501507, 1995 (Japanese).

[8] M. Tohda, H. Hayashi, M. Sukma, and K. Tanaka, "BNIP-3: a novel candidate for an intrinsic depression-related factor found in NG108-15 cells treated with Hochu-ekki-to, a traditional oriental medicine, or typical antidepressants," Neuroscience Research, vol. 62, no. 1, pp. 1-8, 2008.

[9] M. Tohda, S. Mingmalairak, Y. Murakami, and K. Matsumoto, "Enhanced expression of BCL2/adenovirus EIB 19$\mathrm{kDa}$-interacting protein $3 \mathrm{mRNA}$, a candidate for intrinsic depression-related factor, and effects of imipramine in the frontal cortex of stressed mice," Biological and Pharmaceutical Bulletin, vol. 33, no. 1, pp. 53-57, 2010.

[10] M. L. Kram, G. L. Kramer, M. Steciuk, P. J. Ronan, and F. Petty, "Effects of learned helplessness on brain GABA receptors," Neuroscience Research, vol. 38, no. 2, pp. 193-198, 2000.

[11] B. Vollmayr, C. Simonis, S. Weber, P. Gass, and F. Henn, "Reduced cell proliferation in the dentate gyrus is not correlated with the development of learned helplessness," Biological Psychiatry, vol. 54, no. 10, pp. 1035-1040, 2003.

[12] P. Willner, A. Towell, D. Sampson, S. Sophokleous, and R. Muscat, "Reduction of sucrose preference by chronic unpredictable mild stress, and its restoration by a tricyclic antidepressant," Psychopharmacology, vol. 93, no. 3, pp. 358-364, 1987.

[13] S. Ito, Y. Nitta, H. Fukumitsu et al., "Antidepressant-like activity of 10-hydroxy-trans-2-decenoic acid, a unique unsaturated fatty acid of royal jelly, in stress-inducible depression-like mouse model," Evidence-Based Complementary and Alternative Medicine, vol. 2012, Article ID 139140, 6 pages, 2012.

[14] N. Ito, T. Nagai, T. Oikawa, H. Yamada, and T. Hanawa, "Antidepressant-like effect of l-perillaldehyde in stress-induced depression-like model mice through regulation of the olfactory nervous system," Evidence-Based Complementary and Alternative Medicine, vol. 2011, Article ID 512697, 5 pages, 2011.

[15] J. Yamakawa, Y. Motoo, J. Moriya et al., "Role of kampo medicine in integrative cancer therapy," Evidence-Based Complementary and Alternative Medicine, vol. 2013, Article ID 570848, 6 pages, 2013. 


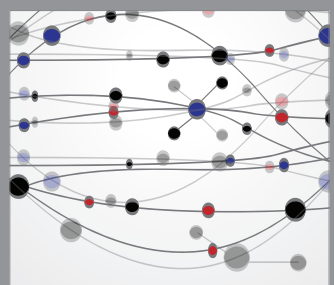

The Scientific World Journal
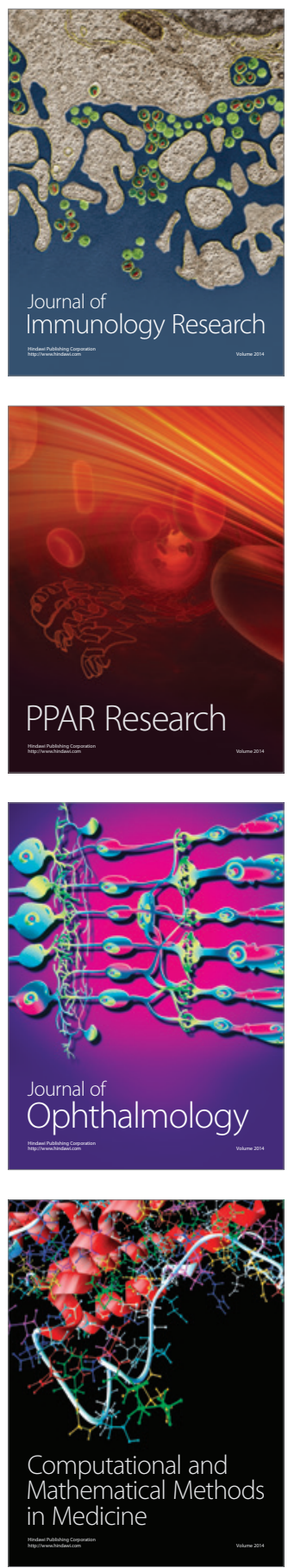

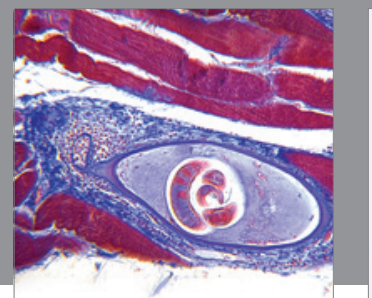

Gastroenterology

Research and Practice
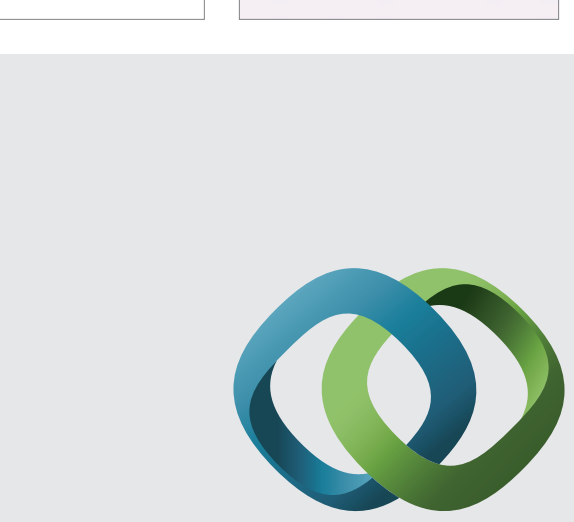

\section{Hindawi}

Submit your manuscripts at

http://www.hindawi.com
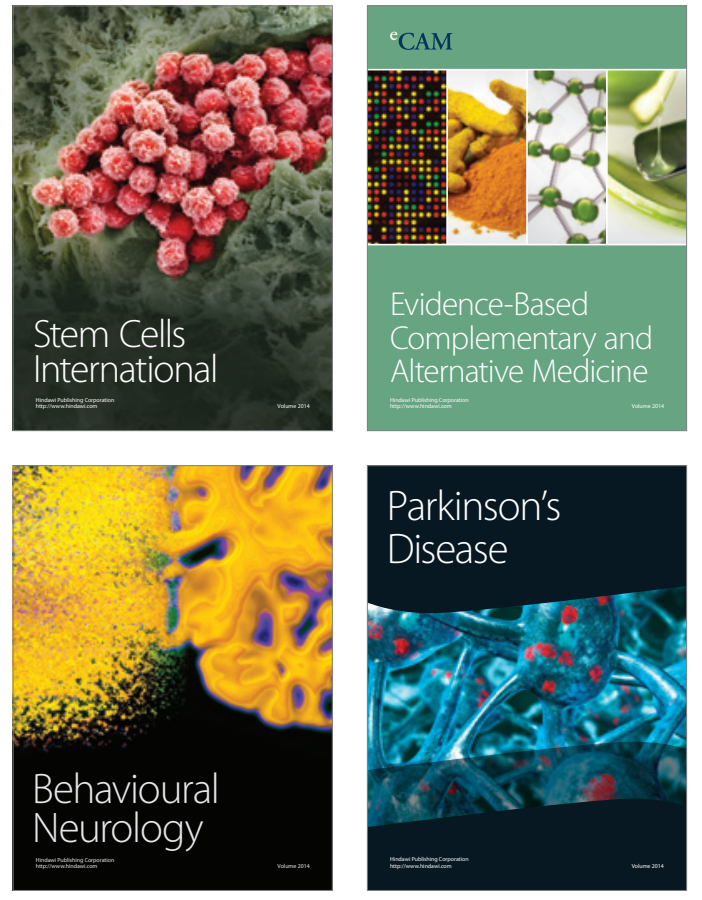
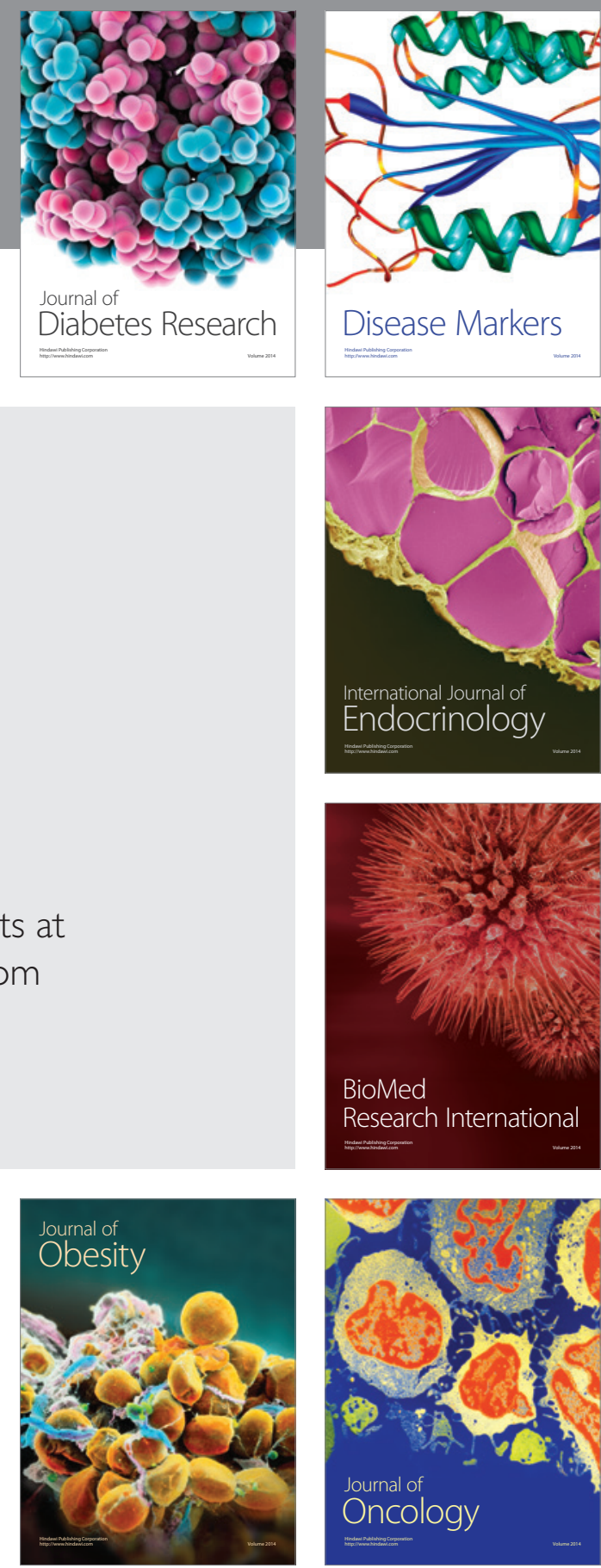

Disease Markers
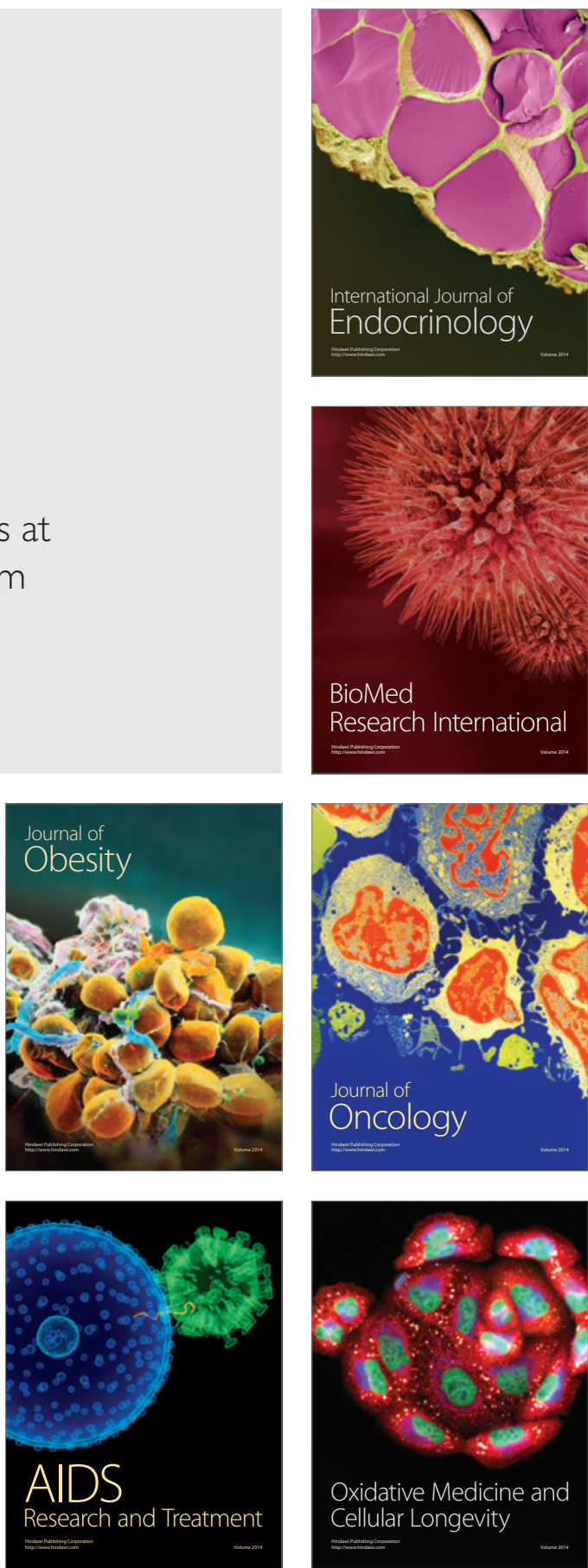